\begin{tabular}{|c|c|c|}
\hline Beitr. Ent. & Keltern & ISSN 0005-805X \\
\hline $\mathbf{5 7}(2007) 2$ & S. $321-333$ & 21.12 .2007 \\
\hline
\end{tabular}

\title{
Beiträge zur Ichneumonidenfauna Deutschlands. 1. Apechthis FÖRSTER, 1869
}

\section{(Hymenoptera, Ichneumonidae, Pimplinae)}

Mit 4 Figuren und 2 Tabellen

HaNS-JOACHIM JaCOBS

\section{Zusammenfassung}

Für die vier in Deutschlands verbreiteten Apechthis-Arten wurden Bestimmungstabellen und Beschreibungen erarbeitet, publizierte faunistische Daten gewertet und diese mit Daten aktuell bestimmten Materials zu einer Checkliste für die deutschen Bundesländer zusammengefasst.

Faunistische Literaturangaben erwiesen sich als nicht in jedem Fall zuverlässig und wurden getrennt von den hier untersuchten Belegexemplaren erfasst. A. compunctor (Linnaeus, 1758), A. quadridentata (Thomson, 1877) und A. rufata (Gmelin, 1790) sind aus allen Bundesländern belegt. Die Mehrzahl der Nachweise stammt aus der Zeit vor 1980, da später nur noch unzureichend Entomofaunistik betrieben wurde. A. capulifera (Kriechbaumer, 1887) ist in Deutschland ebenfalls weit verbreitet aber sehr selten (letzter überprüfter Nachweis von 1935).

\section{Summary}

For the four Apechthis species, which occur in Germany, an identification key and redescriptions are presented. Published faunistic data are evaluated and summarized in a checklist for the German federal states together with newly identified material. Since faunistic data from literature sometimes turned out as not reliable, these have been listed separately. A. compunctor (Linnaeus, 1758), A. quadridentata (Thomson, 1877) and A. rufata (Gmelin, 1790) occur in all German federal states. Most records for these species originate from the period before 1980 since in later years faunistic studies have been conducted only at an insufficient extent. A. capulifera (Kriechbaumer, 1887) is also widely distributed in Germany but it is rare (last hereby checked record from 1935).

\section{Key words}

Hymenoptera, Ichneumonidae, Pimplinae, Apechthis, Fauna, Deutschland.

\section{Einleitung}

Die meisten Familien der parasitoiden Hymenopteren (Schlupfwespen im weitesten Sinne) sind im Verzeichnis der Hautflügler Deutschlands (DATHE et al. 2001) nur in Form einer Checkliste aufgeführt. Die fehlenden Verbreitungsangaben für einzelne Bundesländer sind ein deutlicher Ausdruck mangelnder faunistischer Forschung. Eine Hauptursache hierfür sieht KLAusnitzer (2003) im Fehlen moderner und zugänglicher Bestimmungstabellen. Dieser Zustand wird sich für viele Gruppen parasitischer Hymenopteren in absehbarer Zeit auch nicht ändern, da es für viele dieser Taxa zu wenige oder gar keine Bearbeiter gibt. Die Beiträge in dieser Reihe sollen ein erster Anfang sein, sich faunistisch mit einigen dieser Gruppen zu beschäftigen. 
Die Gattung Apechthis Förster, 1869 ist mit 16 Arten in der Paläarktis, Orientalis, Nearktis und Neotropis vertreten (Yu \& Horstmann 1997, Yu 1999). In der Paläarktis leben fünf Arten, von denen vier in Deutschland nachgewiesen sind (Horstmann 2001). Sie sind in der Waldzone Eurasiens weit verbreitet, nur A. rapae (UchIdA, 1925) ist auf den Fernen Osten beschränkt. Die Unterfamilie ist mit den Schlüsseln von OehlKe (1969) oder KaSparyan (1981) erkennbar, die Gattung nach Oehlke (1964), Kasparyan (1981), Fitton, Shaw \& Gauld (1988) oder Kolarov (1997). Einen Bestimmungsschlüssel für die paläarktischen Arten publizierte KasPaRYAN (1973). Neuere Schlüssel für die europäischen Arten erarbeiteten Kasparyan (1981) und Kolarov (1997, 2004). Obwohl die Arten nach den Schlüsseln von Schmiedeknecht (1907, 1914) durchaus ansprechbar sind, können Fehldeterminationen nicht ausgeschlossen werden. In der vorliegenden Arbeit soll eine Bestimmungstabelle für die in Mitteleuropa lebenden Arten gegeben und ihre Verbreitung in Deutschland dargestellt werden. Basis dafür sind Literaturdaten (in der optimistischen Annahme einer korrekten Determination), der Sammlungsbestand des Deutschen Entomologischen Institutes im ZALF Müncheberg (DEI) und eigene Aufsammlungen, meist aus Mecklenburg-Vorpommern. Wie wichtig Sammlungsrevisionen für die faunistische Arbeit sind, zeigen die Fehldeterminationen im Sammlungsmaterial des DEI (Tab. 1). Besonders häufig wurde A. rufata nicht sicher erkannt. Viele Tiere trugen keine Determinationsetiketten und könnten aus diesem Grunde falsch gesteckt sein. Das wirft natürlich Fragen über die Verwendbarkeit von Angaben auf, die nicht auf modernen Bestimmungstabellen fußen. Nach Horstmann (2002) ist bei Faunenlisten von Ichneumoniden mit einer Fehldeterminationsrate von 15-20 \% zu rechnen, wobei seltene Arten häufiger fehldeterminiert sind als häufige. Deshalb sind Literaturdaten im Text und in der Checkliste (Tab. 2) gesondert ausgewiesen. SMits van Burgst (1918) weist alle vier Arten für Deutschland nach, gibt aber keine Fundorte an. In der faunistischen Literatur verwendete Synonyme sind bei den Arten angegeben. Die vollständige Synonymie ist den Katalogen von Oehlke (1967), Yu \& Horstmann (1997) bzw. Yu (1999) zu entnehmen.

\begin{tabular}{llll}
\multicolumn{2}{c}{ Abkürzungen: } & & \\
$\mathrm{Br}$ & Brandenburg und Berlin & $\mathrm{BW}$ & Baden-Württemberg \\
$\mathrm{By}$ & Bayern & $\mathrm{He}$ & Hessen \\
$\mathrm{MV}$ & Mecklenburg-Vorpommern & $\mathrm{Ni}$ & Niedersachsen und Bremen \\
$\mathrm{NW}$ & Nordrhein-Westfalen & $\mathrm{PA}$ & Punktabstände \\
$\mathrm{PD}$ & Punktdurchmesser & $\mathrm{RP}$ & Rheinland-Pfalz und Saarland \\
$\mathrm{SH}$ & Schleswig-Holstein und Hamburg & $\mathrm{Sn}$ & Sachsen \\
$\mathrm{St}$ & Sachsen-Anhalt & $\mathrm{Th}$ & Thüringen \\
$<$ & kleiner als & $>$ & größer als
\end{tabular}

Tab. 1: Fehldeterminationen im Material des DEI

\begin{tabular}{|l|c|c|c|c|}
\hline Material DEI & $\begin{array}{c}\text { capulifera } \\
\text { (13 Ex.) }\end{array}$ & $\begin{array}{c}\text { compunctor } \\
\text { (91 Ex.) }\end{array}$ & $\begin{array}{c}\text { quadridentata } \\
\text { (82 Ex.) }\end{array}$ & $\begin{array}{c}\text { rufata } \\
\text { (80 Ex.) }\end{array}$ \\
\hline capulifera & 13 & 1 & 0 & 0 \\
\hline compunctor & 0 & 77 & 1 & 4 \\
\hline quadridentata & 0 & 6 & 79 & 28 \\
\hline rufata & 0 & 2 & 0 & 41 \\
\hline andere Gattungen & 0 & 5 & 2 & 7 \\
\hline
\end{tabular}


Tab. 2: Nachweise in Bundesländern ( $\square$ Literaturnachweis vor 1980, - Literaturnachweis ab 1980, ? fraglicher Literaturnachweis, o kontrollierter Beleg vor 1980, • kontrollierter Beleg ab 1980)

\begin{tabular}{|l|c|c|c|c|c|c|c|c|c|c|c|c|}
\hline Art/Bundesl. & $\mathrm{SH}$ & $\mathrm{MV}$ & $\mathrm{Ni}$ & $\mathrm{St}$ & $\mathrm{Br}$ & $\mathrm{NW}$ & $\mathrm{He}$ & $\mathrm{Th}$ & $\mathrm{Sn}$ & $\mathrm{RP}$ & $\mathrm{BW}$ & $\mathrm{By}$ \\
\hline capulifera & & $\circ$ & $\square$ & $\square$ & $\circ$ & $\square$ & & & ? & & & $\square$ \\
\hline compunctor & $\circ$ & $\bullet$ & $\circ$ & $\bullet$ & $\bullet$ & $\circ$ & $\circ$ & $\bullet$ & $\mathbf{\square}$ & $\square$ & $\square$ & $\circ$ \\
\hline quadridentata & $\circ$ & $\bullet$ & $\square$ & $\circ$ & $\bullet$ & $\square$ & $\circ$ & $\circ$ & $\circ$ & $\square$ & $\square$ & $\square$ \\
\hline rufata & $\square$ & $\bullet$ & $\square$ & $\square$ & $\circ$ & $\square$ & $\circ$ & $\circ$ & $\square$ & $\square$ & $\square$ & $\square$ \\
\hline
\end{tabular}

\section{Lebensweise}

Alle Arten der Gattung Apechthis sind Puppenparasiten bei einer Vielzahl von Schmetterlingen (Lepidoptera) aus verschiedensten Familien. Die Eiablage erfolgt direkt in die Wirtspuppe. Cole (1959) deutet die gebogene Spitze des Legebohrers als Anpassung an die Belegung von Schmetterlingspuppen durch die Intersegmentalhaut. Das erfordert freie Puppen bzw. solche in dünnen Blattwickeln. Harte und glatte Puppen werden deutlich weniger belegt. Die Wirtsspektren der einzelnen Arten unterscheiden sich nicht wesentlich. Yu (1999) listet als Wirte Lepidoptera aus folgenden Familien: Arctiidae, Drepanidae, Gelechiidae, Geometridae, Hesperiidae, Lasiocampidae, Lycaenidae, Lymantriidae, Noctuidae, Nymphalidae, Oecophoridae, Papilionidae, Pieridae, Psychidae, Pyralidae, Saturniidae, Tortricidae und Zygaenidae. Wirtsarten und zugehörige Zitate sind den Katalogen von Aubert (1969), Yu \& Horstmann (1997) bzw. Yu (1999) zu entnehmen. Bevorzugt werden Schmetterlingsarten, die sich in der Vegetation oder in Bodennähe verpuppen. Puppen im Boden dürften für Apechthis unerreichbar sein, da die Weibchen nicht graben können. Deshalb sind Wirtsangaben von Sphingidae für $A$. compunctor (Dalla Torre 1902, Rudow 1917) und A. rufata (Drewsen \& BoIE 1836) zweifelhaft. Alte Angaben bedürfen generell der Überprüfung (siehe auch Aubert 1969). Daneben gibt es einzelne Berichte über die Parasitierung von Insekten anderer Ordnungen und von Spinnen (Arachnida). A. quadridentata und A. rufata sollen danach auch Kokons von Blattwespen (Diprionidae) parasitieren (GYörFI 1963, Š EDIvÝ 1963). Eine Zucht aus Cephalcia abietis (Linnaeus, 1758), von der Šedivý (1963) berichtet, beruht möglicherweise auf einem Irrtum, da da sich Pamphiliidae im Boden verpuppen (Enslin 1912-1918). Die Parasitierung von Gallen des Bockkäfers Saperda populnea (L.) durch A. capulifera (Schütze \& Roman 1931, Starke 1940, 1956, Uhthoff-Kaufmann 1991) ist ebenfalls unwahrscheinlich. Der relativ kurze, mit der Spitze nach unten gebogene Legebohrer eignet sich kaum, um dicke und verholzte Gallenwände zu durchdringen. UHTHOFF-KaUfmanN (1991) veröffentlichte eine kommentarlose Parasitoidenliste, aus der nicht hervorgeht, ob er eine der genannten Arten selbst gezogen hat. A. capulifera wurde in Großbritannien bisher nicht nachgewiesen. Unter Laborbedingungen parasitierten $A$. compunctor und $A$. rufata auch Puppen des Mehlkäfers Tenebrio molitor (Linnaeus, 1758) (Aubert 1959), der aber mit Sicherheit ebenfalls nicht zum natürlichen Wirtsspektrum zählen dürfte.

In der Literatur hielt sich lange Zeit die Angabe von Bouché (1834), der A. rufata aus dem Eikokon von Aranea diadema Linnaeus, 1758 gezogen haben will. Hier lag wahrscheinlich eine Verwechslung mit Arten der Gattungen Tromatobia Förster, 1869 oder Zaglyptus Förster, 1869 vor.

Die Habitatansprüche der Apechthis-Arten sind relativ einheitlich. Sie bewohnen lichte Laubund Mischwälder mit reichlichem Unterwuchs, deren Ränder, Parks, Gärten und Gebüsche. 
Auf die besondere Bedeutung eines reichhaltigen Unterwuchses in Wäldern für eine artenreiche Ichneumonidenfauna weisen GYörfi (1963) und SAwOnIEwiCz (1979) hin. Die Flugzeit der Imagines erstreckt sich in Abhängigkeit von der Witterung von Ende April bis Ende Oktober.

\section{Bestimmungstabelle der Arten}

$1 \quad$ Hinterhüften ganz und Hinterschenkel apikal schwarz. Mesoscutum schwarz, \pm lederartig matt. 9-18 mm. Apechthis capulifera (KRIECHBAUMer, 1887)

1* Hinterhüften und -femora rot. Mesoscutum \pm glänzend. 2

2 우우. 3 $0^{\top} 0^{\top}$.

3 Hinterklauen einfach, ohne Basalzahn (Fig. 1). 6-15 mm

4 Tergum 1 oben am Knick mit deutlichen Höckern (Fig. 3). Hintertibien einfarbig rot. Mesoscutum schwarz. Gelbe Zeichnung entlang der inneren Orbiten nicht über die Augenausrandung hinausgehend. Propodeum braun behaart. 8-17 mm.

Apechthis compunctor (LinNaEus, 1758)

4* Tergum 1 oben am Knick mit schwachen Höckern (Fig. 4). Hintertibien proximal dunkelbraun, distal oben \pm dunkelbraun, unten rotbraun, mit weißem Subbasalring. Mesoscutum wenigstens in der hinteren Hälfte mit zwei gelben Streifen entlang der Notauli. Gelbe Zeichnung entlang der inneren Orbiten bis auf den Scheitel reichend. Propodeum weißgrau behaart. 6-15 $\mathrm{mm}$.

Apechthis rufata (GMELIN, 1790)

5 Gesicht unter den Fühlern mit \pm großem schwarzen Fleck. Hintertibien rot, apikal \pm verdunkelt. Propodeum braun behaart.

Apechthis compunctor (LinnaEus, 1758)

Gesicht vollständig gelb. Hintertibien schwarz mit weißem Subbasalring. Propodeum weißgrau behaart. 6

6 Terga 5-7 im distalen Drittel deutlich und fast so dicht punktiert wie im proximalen Teil, Oberfläche rau und matt. Mesoscutum schwarz, in der Mitte meist mit zwei durchgehenden gelben Strichen entlang der Notauli, Vorderrand zuweilen \pm gelb gefärbt.

Apechthis rufata (GMELIN, 1790)

Terga 5-7 im distalen Drittel deutlich feiner und zerstreuter punktiert als im proximalen Teil, Oberfläche glatt und glänzend. Mesoscutum schwarz, meist ohne gelbe Zeichnung, manchmal am Vorderrand mit zwei kleinen gelben Makeln oder in der Mitte mit zwei kurzen und dünnen, aber niemals durchlaufenden Längsstreifen.

Apechthis quadridentata (ТномsоN, 1877) 

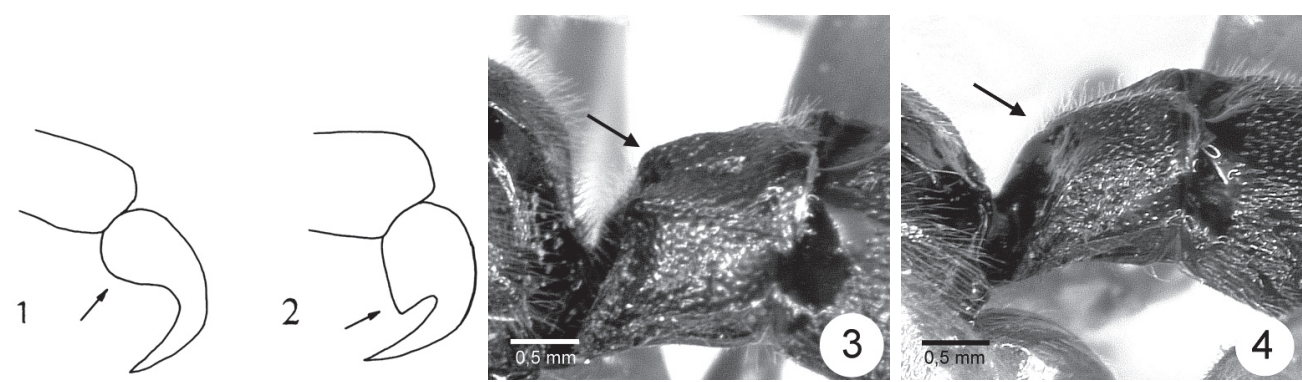

Fig. 1-4: 1 Hinterklaue $A$. quadridentata ․ - 2: Hinterklaue $A$. compunctor 우. - 3: Tergum 1 A. compunctor ㅇ․ - 4: Tergum 1 A. rufata ㅇ․

\section{Verzeichnis der Arten}

\section{Apechthis capulifera (KRIECHBAUMER, 1887)}

\section{Beschreibung:}

ㅇ 우 : Körper ohne Legebohrer 11,5-18,5 mm, Vorderflügel 9,5-14 mm. Fühler: Scapus schwarz. Pedicellus schwarz, unten rot. Geißel rotbraun, oben \pm verdunkelt. Kopf: Schwarz. Gelb sind schmale Streifen an den inneren Orbiten, die meist nicht über die Augenausrandung über der Fühlereinlenkung hinausgehen und manchmal winzige Scheitelflecken. Integument unpunktiert, \pm matt. Mesosoma: Schwarz. Gelb sind Flecken an den Hinterrändern von Scutellum und Postscutellum. Mesonotum unpunktiert, lederartig matt, übrige Teile des Mesosoma \pm glänzend. Mesopleuren deutlich, aber zerstreut punktiert $(\mathrm{PA}>\mathrm{PD})$. Propodeum dicht punktiert $(\mathrm{PA}<\mathrm{PD})$. Tegulae schwarz. Metasoma: Schwarz. Terga 1-5 dicht und grob punktiert (PA>PD). Tergum 6 feiner punktiert als die vorhergehenden, 7 fast unpunktiert. Terga \pm matt. Vorderbein: Coxa schwarz, manchmal gelb gefleckt. Restliches Bein rot mit \pm gelben Anteilen. Trochanter $1 \pm$ verdunkelt. Mittelbein: Coxa und Trochanter 1 schwarz, restliches Bein \pm rot. Hinterbein: Coxa und Trochanter 1 schwarz, Trochanter 2 rot, unten \pm verdunkelt. Tibien dunkelbraun, mit weißem Subbasalring. Tarsus dunkelbraun.

$o^{\star} o^{*}$ : Körper ca. $15 \mathrm{~mm}$, Vorderflügel 11,5 mm. Fühler: Scapus und Pedicellus schwarz, unten gelb. Geißel rotbraun. Kopf: Schwarz. Gelb sind Maxillar- und Mandibularpalpus, Mandibelbasis, Labrum, Clypeus, Gesicht unter der Fühlereinlenkung bis auf einen schwarzen Längsstreifen in der Mitte, Streifen an den inneren Orbiten der Stirn und Scheitelflecke. Mesosoma: Schwarz. Gelb sind Tegulae sowie die Hinterränder von Scutellum und Postscutellum, sehr selten mit zwei kurzen gelben Längslinien (Habermehl 1917). Rot sind die Hinterecken des Pronotums vor den Tegulae, der Längswulst im oberen Teil der Mesopleuren unter dem Ansatz des Vorderflügels. Mesoscutum unpunktiert, lederartig matt. Propodeum dicht und deutlich punktiert $(\mathrm{PA}<\mathrm{PD})$. Mesopleuren fein und zerstreut punktiert $(\mathrm{PA}>\mathrm{PD})$. Integument außer dem Mesoscutum glänzend. Metasoma: Terga grob und dicht punktiert $(\mathrm{PA}<\mathrm{PD})$. Vorderbein: Coxa schwarz, vorn \pm gelb gefleckt. Trochanteren, Femora und Tibien vorn gelb, hinten \pm rot. Tarsus gelbrot. Mittelbein: Coxa schwarz, restliches Bein rot, Femora und Tibien vorn \pm gelb. Hinterbein: Coxa schwarz. Trochanteren rot, \pm verdunkelt. Femora rot, apikal verdunkelt. Tibien dunkelbraun mit weißem Subbasalring. Tarsus dunkelbraun.

Literaturangaben für Deutschland: Br: Berlin (BRAuns 1898), Fürstenberg (Anmerkung von Konow bei Bengtsson 1902). By: Füssen (loc. typ.) (Kriechbaumer 1887). He: Harreshausen (Habermehl 1917). 
MV: "Überall in Mecklenburg" (Brauns 1898). Ni: Hasbruch (Pfankuch 1935). NW: Dahlheim (Ulbricht 1926, Aerts 1957). Sn: Löbau (Starke 1956) (Meldung fraglich, da Wirtsangaben mit Sicherheit falsch, Verwechslung oder Fehldetermination). St: Halle (НAupt 1913).

Untersuchtes Material (3 $\sigma^{\star} \sigma^{\star}, 10$ ㅇ ㅇ): Br: Berlin-Köpenick, Rangnow (ANONYM), Fürstenberg (Konow), Tiefensee (Lichtwardt), Finkenkrug (Bollow), Dannenberg bei Berlin (Lüdeke). MV: Warnemünde (KoNOw), Bansin (LÜDEKE).

\section{Verbreitung:}

Paläarktis mit Verbreitungsgrenzen im westlichen Mitteleuropa (Deutschland, Schweiz) und Südosteuropa, offenbar nicht im ausgeprägt atlantischen und mediterranen Bereich. Nach KASPARYAN (1981) in der Ostpaläarktis häufiger. A. capulifera ist in Deutschland sehr selten. Der letzte geprüfte Nachweis stammt von 1935, die letzte glaubhafte Angabe aus der Literatur von 1925 (Ulbricht 1926). Aerts (1957) bezieht sich wahrscheinlich auf diesen Nachweis.

\section{Apechthis compunctor (Linnaeus, 1758)}

Synonyme in den Literaturangaben: Apechthis brassicariae (PoDA, 1761), Apechtis brassicariae (PoDA, 1761), Ephialtes compunctor (Linnaeus, 1758), Pimpla brassicariae (Poda, 1761).

\section{Beschreibung:}

ㅇ 우: Körper 10-15 mm, Vorderflügel 7,5-12 mm. Fühler: Scapus schwarz. Pedicellus schwarz, unten gelbrot. Geißelglied 1 oben schwarz, unten gelb. Restliche Geißel rotbraun, \pm verdunkelt. Kopf: Schwarz. Innere Orbiten schwarz oder mit \pm ausgedehntem gelben Streifen, der die Augenausrandung meist nicht überschreitet. Integument unpunktiert, glänzend. Maxillarpalpen bis auf die beiden schwarzen Basalglieder rotbraun. Labialpalpen dunkelbraun bis schwarz. Mesosoma: Schwarz, nur Hinterränder von Scutellum und Postscutellum mit gelben Flecken. Tegula zuweilen am Außenrand rötlich aufgehellt. Integument stark glänzend, bis auf das Propodeum fast unpunktiert. Metasoma: Schwarz. Terga 1-5 bis auf den eingedrückten Hinterrand dicht und grob punktiert $(\mathrm{PA}<\mathrm{PD})$. Terga 6-7 glatter, feiner und zerstreuter punktiert als die vorherigen. Vorderbein: Coxa schwarz, apikal rotgelb. Restliches Bein rot, Femora und Tibien vorn gelb. Mittelbein: Rot, Coxa basal \pm schwarz. Femur apikal vorn mit kleinem gelbem Fleck. Tibia zuweilen vorn gelb. Hinterbein: Rot, Tarsomere 3-5 \pm verdunkelt. Tibien manchmal mit der Andeutung eines helleren Subbasalringes.

$o^{\star} o^{\star}$ : Körper 9,5-15 mm, Vorderflügel 6,5-10,5 mm. Fühler: Scapus und Pedicellus unten gelb. Geißel oben dunkelbraun, unten gelbrot. Kopf: Schwarz. Gelb sind ein Fleck auf dem Labrum, Clypeus ganz, Gesicht bis auf einen \pm großen schwarzen Mittelstreifen, innere Orbiten über den Fühlereinlenkungen, Scheitelflecken, oft Mandibelbasis. Die gelbe Gesichtsfärbung kann reduziert sein bis auf einen schmalen Orbitalstreifen. Integument unpunktiert und glänzend. Mesosoma: Schwarz. Gelb sind zwei Streifen in der Mitte des Mesoscutums (oft fehlend), Tegula (die auch rot sein kann), ein Fleck auf den Mesopleuren unter dem Vorderflügelansatz sowie die Hinterränder von Scutellum und Postscutellum. Rötlich ist die Hinterecke des Pronotums vor den Tegulae. Propodeum dicht und grob punktiert, mit Ausnahme des Mittelfeldes. Übriges Integument kaum punktiert, glatt und glänzend. Metasoma: Terga 1-6 grob und dicht punktiert $(\mathrm{PA}<\mathrm{PD})$. Tergum 7 in ganzer Länge punktiert, aber weniger dicht und grob als das davor. Vorderbein: Coxa schwarz, apikal \pm gelb, hinten rot oder nur schwarz und rot. Trochanter 1 gelb, 2 rot. Femur rot, vorn gelb. Tibia gelbrot, Tarsus rot. Mittelbein: Coxa rot, manchmal vorn gelb, basal mit schwarzem Fleck. Trochanteren rot. Femur rot, vorn \pm gelb. Tibia rot, vorn \pm gelb oder ganz gelbrot. Tarsus gelbrot. Hinterbein: Rot. Tibia zuweilen basal etwas verdunkelt, manchmal mit sehr schwach angedeutetem hellem Subbasalring. Tarsus ab Spitze des Metatarsus \pm verdunkelt. 
Literaturangaben für Deutschland: Br: Berlin-Spandau (Ulbricht 1912), Berlin-Dahlem (Strand 1918). BW: Wildbad im Schwarzwald (Pfeffer 1913), Ladenburg (Haeselbarth 1985), Feldberg im Schwarzwald (Hilpert 1987a, b), Weisweil (Hilpert 1989). By: Berchtesgaden (Heinrich 1949), NürnbergErlangen (Bauer 1958), Schraudenbach (Horstmann 1971), Wiesen/Spessart (Haeselbarth 1985). Ni: Bremen, Stotel, Wollah (Pfankuch 1935), Gifhorn/Eyßelheide (Hedwig 1958), Berel (Hedwig 1959), Bremerhaven, Flögeln (Kettner 1968), Münden (Haeselbarth 1985). NW: Krefeld (Ulbricht 1909), Bergisch Gladbach, Hückeswagen, Köln, Krefeld, Rösrath (Aerts 1957). RP: Worms (Habermehl 1904, 1917), Burgen/Mosel (Aerts 1957). SH: Hamburg (KettNer 1968). Sn: Sächsische Oberlausitz verbreitet, Göda (Starke 1956), Altenberg (Walter 1991). St: Halle (Haupt 1913, 1954). Th: Erfurt, Gotha, Tabarz, Windleite (JäNNER 1919a).

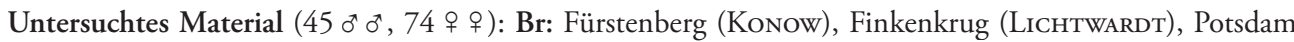
(Bollow), Berlin (GärTner, LÜDeke), Berlin-Steglitz, Strausberg (LÜDeke), Eberswalde, Melchow, Störitzsee (OehlKe), Brodowin (Stahl). By: Elmau (AnONym), Oberstdorf (LÜdeke). He: Groß Karben (Leonardi). MV: Bansin (ANonym), Kalkhorst, Teschendorf, Warnemünde (Konow), Müritzhof (Oenlke), Boldekow, Dambeck, Demmin, Groß Kiesow, Gützkow, Hohendorf, Jakobsdorf, Karlsburg, Landskron, Lüssow, Oldenburg, Paske, Pentin, Ranzin, Rothemühl, Schmatzin, Wrangelsburg (JaCOBS). Ni: Niederhaverbeck (JÄ́кh). NW: Detmold (Pape), Krefeld (Ulbricht). SH: Hamburg-Fischbek (Anonym), HamburgBramfeld, Hamburg-Neugraben (LÜdeke), Archsum, Sylt (Ohaus). Sn: Dresden (Anonym), Saubachtal (Oehlke). St: Halle (Anonym), Ilfeld/Südharz (TAeger \& TAeger). Th: Erfurt (Thust).

\section{Verbreitung:}

Paläarktis außer Nordafrika und Naher Osten. In Deutschland die häufigste Art der Gattung. In den Alpen bis in Höhen von ca. 2300 m (BAuer 2002).

\section{Apechthis quadridentata (ThомsоN, 1877)}

Synonyme in den Literaturangaben: Apechthis resinator (Thunberg, 1822), Apechtis resinator (Thunberg, 1822), Ephialtes quadridentata (Thомson, 1877), Ephialtes quadridentatus (Thomson, 1877), Pimpla quadridentata Thomson, 1877, Pimpla resinator (Thunberg, 1822).

\section{Beschreibung:}

ㅇ 오 : Körper 7-15 mm, Vorderflügel 6,5-12,5 mm. Fühler: Scapus und Pedicellus meist schwarz, letzterer unten manchmal gelb. Geißel oben dunkelbraun, unten rötlich aufgehellt. Kopf: Schwarz. Gelb sind ein schmaler Streifen an den inneren Orbiten und ein Scheitelfleck. Palpen rotgelb. Integument unpunktiert, glatt und glänzend. Mesosoma: Schwarz. Gelb sind die Hinterränder von Scutellum und Postscutellum. Rötlich ist der Hinterrand des Pronotums vor den Tegulae. Mesoscutum kaum punktiert, Mesopleuren und Scutellum fein und zerstreut punktiert, Propodeum außer dem Mittelfeld dicht und grob punktiert. Integument insgesamt glänzend. Metasoma: Schwarz. Terga 1-5 außer an den eingedrückten Hinterrändern dicht punktiert $(\mathrm{PA}<\mathrm{PD})$. Tergum 6 nur in der Basalhälfte punktiert, apikal \pm glatt. Tergum 7 nur auf dem Basalteil fein punktiert, auf dem Rest unpunktiert. Vorderbein: Coxa basal schwarz, apikal rot. Bein \pm rot, Femur und Tibia vorn \pm gelb. Mittelbein: Rot. Femur apikal mit kleinem gelbem Fleck, Tibia basal \pm gelblich. Hinterbein: Rot. Tibia mit dunkelbrauner Basis und weißem Subbasalring, in der distalen Hälfte rot oder braun. Metatarsus basal weißlich aufgehellt. Restlicher Tarsus rot oder bräunlich.

$o^{\star} o^{\star}$ : Körper 7-12 mm, Vorderflügel 6-9 mm. Fühler: Scapus und Pedicellus schwarz, unten gelb. Geißel oben dunkelbraun bis schwarz, unten rötlich aufgehellt. Kopf: Schwarz. Gelb sind Palpen, Clypeus, Gesicht bis unter die Fühlereinlenkungen, ein Streifen an den inneren Orbiten im Bereich der Stirn und auf dem Scheitel. Integument fast unpunktiert, glatt und 
glänzend. Mesosoma: Schwarz. Gelb sind die Hinterecken des Pronotums, Tegulae, ein Fleck auf den Mesopleuren unter den Flügeln, manchmal zwei Flecken oder Streifen am Vorderrand des Mesoscutums bei den Notauli sowie die Hinterränder von Scutellum und Postscutellum. Propodeum bis auf das Mittelfeld grob punktiert. Übriges Integument höchstens sehr vereinzelt und fein punktiert, glatt und glänzend. Metasoma: Schwarz. Terga 1-5 außer auf den wulstigen Hinterrändern grob und dicht punktiert $(\mathrm{PA}<\mathrm{PD})$. Terga 5-6 an der Basis schwächer punktiert als die vorigen, im apikalen Drittel glatt und fast unpunktiert. Vorderbein: Coxa ganz gelb oder mit roten Anteilen. Trochanteren gelb. Restliches Bein meist rötlichgelb, Tibia und Metatarsus zuweilen etwas heller. Femur und Tibia zuweilen vorn gelb. Mittelbein: Coxa gelb oder \pm rot. Trochanteren gelb. Übriges Bein rot, Femur, Tibia und Metatarsus unten \pm gelb. Tibia und Tarsus können auch gelb sein. Hinterbein: Coxa rot, Trochanteren gelb. Femur rot. Tibia im apikalen Drittel rot oder braun, basal dunkelbraun, mit weißem Subbasalring. Tarsomere 1-2 basal hell, apikal braun. Tarsomere 3-5 braun.

Literaturangaben für Deutschland: Br: Berlin, Potsdam (Ulbricht 1912). BW: Wildbad im Schwarzwald (Pfeffer 1913), Hinterzarten (Hilpert 1987a), Weisweil (Hilpert 1989). By: Berchtesgaden (Heinrich 1949), Nürnberg-Erlangen (Bauer 1958), Schraudenbach (Horstmann 1971), Schweinfurth (Horstmann 1984), Bad Neustadt/Saale (Haeselbarth 1985), Limbach/Forstamt Eltmann (Horstmann \& Floren 2001). Ni: Bremen, Syke, Wollah (Pfankuch 1935), Berel, Nordassel (Hedwig 1959), Bremerhaven, Celle (Kettner 1968), Bramwald bei Göttingen, Lehre bei Hannover (Betz \& Schwerdtfeger 1970), Münden (Haeselbarth 1985), Memmert (Horstmann 1988). NW: Krefeld (Ulbricht 1909), Bergisch Gladbach, Hückeswagen, Köln, Krefeld (Aerts 1957). RP: Worms (Habermehl 1917), Daun (Aerts 1957). SH: Hamburg, Trittau (Kettner 1968). Sn: Bautzen, Göda, Neudorf, Königswartha (Starke 1940), Quoos, Bernstadt (Starke 1956), Altenberg, Bärenstein (Walter 1991). St: Halle (Haupt 1913). Th: Thüringen (ohne Ortsangabe) (Schmiedeknecht 1907), Erfurt, Ilmenau (JäNNER 1919b).

Untersuchtes Material: (32 $o^{\star} o^{\star}, 96$ 9 o ): Br: Berlin-Pichelsberg, Potsdam, Wernsdorf, (ANonym), Fürstenberg, (Konow), Berlin (Schwarz, Lichtwardt), Ferch (Schenkling), Sauen (Oehlke \& Petersen), Großmachnow (Schulze), Strausberg (Lüdeke, Oehlke), Luisenfelde (Jacobs). By: Berchtesgaden, Oberstdorf (LüDeKe), Würzburg (ZweCKer), Erlangen (R. BAuer). He: Wiesbaden (Konow). MV: Rügen, Teschendorf (Konow), Bansin (Anonym), Prerow (Oenlke), Boldekow, Hohendorf, Karlsburg, Oldenburg, Ranzin, Wrangelsburg, Züssow (JacoBs). NW: Krefeld (Ulbricht). SH: Hamburg-Fuhlsbüttel (ANONYM), Hamburg-Fischbek (Lüdeke). Sn: Dresden (Konow), Bautzen (Krieger), Leipzig (Riedel, Müller, Oehlke). St: Dölauer Heide (Anonym), Dessau (Krieger). Th: Kyffhäuser (Oehlke).

\section{Verbreitung:}

Paläarktis außer Nordafrika und Naher Osten. In ganz Deutschland verbreitet. Überall im Laubwaldbereich (BAUER 2002).

\section{Apechthis rufata (GMELIN, 1790)}

Synonyme in den Literaturangaben: Apechtis rufata (GMELIN, 1790), Ephialtes rufatus (GMELIN, 1790), Pimpla flavonotata Holmgren, 1860, Pimpla rufata (Gmelin, 1790).

\section{Beschreibung:}

ㅇ 우 : Körper 8,5-16 mm, Vorderflügel 7-12 mm. Fühler: Scapus schwarz. Pedicellus schwarz, unten gelb. Fühlerglied 3 unten gelbrot, oben dunkelbraun. Restliche Geißel rotbraun, unten etwas heller. Kopf: Schwarz. Gelb sind Maxillarpalpen, Labrum, ein schmaler Streifen an den inneren Orbiten bis auf die Stirn und Scheitelflecken. Integument glatt und glänzend. Mesosoma: Schwarz. Gelb sind auf dem Mesoscutum am Vorderrand Streifen zwischen Tegula und Notaulus, 
zwei Streifen an Stelle der Notauli in der hinteren Hälfte des Mesoscutums, Flecken und Streifen am Hinterrand von Scutellum und Postscutellum und manchmal ein kleiner Fleck auf den Mesopleuren unterhalb des Vorderflügels. Rotgelb oder gelb sind Streifen am Hinterrand des Pronotums vor den Tegulae. Integument glatt, bis auf das Propodeum kaum punktiert, glänzend. Tegula rot oder gelb. Metasoma: Schwarz. Terga 1-5 bis auf die unpunktierten Hinterränder grob und dicht punktiert $(\mathrm{PA}<\mathrm{PD})$. Terga 6-7 fein und zerstreut punktiert $(\mathrm{PA}>\mathrm{PD})$. Vorderbein: Coxa rot, basal schwarz. Restliches Vorderbein rot, Trochanteren, Femora und Tibien vorn \pm gelb. Mittelbein: Rot, Femora apikal mit kleinem gelbem Fleck. Hinterbein: Coxa, Trochanteren und Femur rot. Tibia rot, basal schwarz, mit weißem Subbasalring, apikal \pm verdunkelt. Metatarsus rot, apikal \pm verdunkelt. Tarsomere $2-5 \pm$ verdunkelt.

$\sigma^{\star} \sigma^{\star}$ : Körper 8-14 mm, Vorderflügel 6-9 mm. Fühler: Scapus und Pedicellus gelb, oben schwarz. Geißel oben dunkelbraun, die ersten zwei Glieder unten gelb, die folgenden \pm gelbrot bis rot.

Kopf: Schwarz. Gelb sind Labial- und Maxillarpalpen, Labrum, Clypeus, Gesicht bis an die Fühlereinlenkungen, Mandibelbasis, breite Streifen an den oberen inneren Orbiten, die sich oft pfeilförmig bis vor den vorderen Ocellus ausdehnen, Scheitelflecken, zuweilen auch Wangen und unterer Teil der Schläfen. Integument glatt und nahezu unpunktiert. Mesosoma: Schwarz. Gelb sind die hinteren Ecken des Pronotums vor den Tegulae, der Vorderrand des Mesoscutums zwischen Tegula und Notaulus, zwei meistens durchgehende Streifen an der Stelle der Notauli, Tegula, zuweilen Mesopleuren ventral und ein Fleck vorn auf den Epimeren, ein Fleck auf den Mesopleuren unterhalb der Vorderflügel, Flecken am Hinterrand von Scutellum und Postscutellum. Die gelbe Zeichnung der Mesopleuren ist sehr unterschiedlich ausgeprägt und kann fast völlig reduziert sein. Propodeum außer dem Mittelfeld grob und dicht punktiert, die übrigen Teile des Mesosoma glatt und unpunktiert. Integument insgesamt stark glänzend. Metasoma: Schwarz. Alle Terga grob und dicht punktiert, die beiden letzten zerstreuter als die vorhergehenden. Vorderbein: Gelb mit \pm großen roten Anteilen oder überwiegend rot. Mittelbein: Gelb, Femora unten und hinten \pm rötlich. Hinterbein: Coxa und Femur rot. Trochanteren gelb oder rot. Tibia rot mit brauner Basis und weißem Subbasalring, apikal \pm verdunkelt. Tarsomere hellgelb, apikal braun.

Literaturangaben für Deutschland: Br: Finkenkrug (UlbRicht 1912). BW: Wildbad im Schwarzwald (Pfefferer 1913), Weisweil (Hilpert 1989). By: Würzburg (Hedwig 1950), Nürnberg-Erlangen (Bauer 1958), Schraudenbach (Horstmann 1971), Schweinfurth (Horstmann 1984), Limbach/Forstamt Eltmann (Horstmann \& Floren 2001). He: Michelstadt (Habermehl 1904), Marburg (Strand 1913). Ni: Bremen (Pғankuch 1925, 1935), Bassen, Etelsen, Heidkrug, Immer, Memmert, Oldenbüttel, Stotel, Stühe, Wollah (Pfankuch 1935), Gifhorn/Eyßelheide (Hedwig 1958), Nordassel (Hedwig 1959), Borstel, Bremerhaven, Celle, Flögeln, Gifhorn, Sottorf (Kettrner 1968), Bramwald bei Göttingen (Betz \& Schwerdtfeger 1970). NW: Krefeld (Ulbricht 1909), Bergisch Gladbach, Bonn, Köln, Krefeld, Rösrath, Siegburg (Aerts 1957). RP: Worms (Habermehl 1917), Gerolstein, Stromberg/Hunsrück (AerTs 1957). SH: Kiel (Drewsen \& Boie 1836), Großhansdorf, Hamburg, Trittau (KetTner 1968), Niebüll (Horstmann 1970). Sn: Annaberg (Lange 1898), Sächsische Oberlausitz ohne Ortsangabe (STARKe 1956), Altenberg, Bärenstein (Walter 1991). St: Halle (Haupt 1913). Th: Blankenburg, Erfurt, Georgenthal, Gotha, Schwarza, Sondra, Tabarz, Tambach, Windleite (Jänner 1919b).

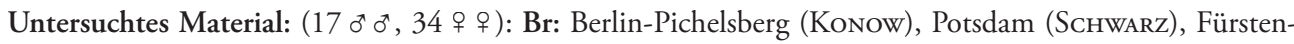
berg (Konow), Berlin-Grunewald (Lichtwardt, Bollow), Finkenkrug (Lichtwardt), Berlin (Lüdeke), Eberswalde (Schieferdecker). BW: Lörrach (LÜdeke). By: Bad Tölz (Lüdeke), Würzburg (Zwecker). He: Karben (Konow). MV: Herthasee, Teschendorf (Konow), Müritzhof (OehlKe), Groß Kiesow, Rustwerder auf Poel (Jacobs). Ni: Münden (Haeselbarth 1985). NW: Krefeld (Anonym). St: Döberitzer Heide (Anonym). Th: Kyffhäuser (OehlKe). 


\section{Verbreitung:}

Paläarktis (außer Nordafrika und Naher Osten), Pakistan, China. In ganz Deutschland, aber seltener als die beiden vorhergehenden Arten. In den Alpen bis in Höhen von ca. 2300 m (BAuer 2002).

\section{Danksagung}

Mein herzlicher Dank gilt Herrn Dr. A. Taeger (Müncheberg) für die Ausleihe des Sammlungsmaterials, Herrn Dr. S. M. Blank (Müncheberg) für die Hilfe bei der Beschaffung der Literatur sowie beiden für die kritische Durchsicht des Manuskriptes.

\section{Literatur}

Aerts, W. 1957: Die Schlupfwespen- (Ichneumoniden-) Fauna des Rheinlandes. - Decheniana 109: 137-212.

Aubert, J. F. 1959: Les hôtes et les stades immatures des Ichneumonides Pimpla F., Apechthis Först. et Itoplectis Först. - Bulletin Biologique de la France et de la Belgique 93: 235-259.

Aubert, J. F. 1969: Les Ichneumonides ouest-paléarctiques et leurs hôtes 1. Pimplinae, Xoridinae, Acaenitinae. - Laboratoire d'Evolution des Etres Organises, Paris: 302 S.

BAUER, R. 1958: Ichneumoniden aus Franken (Hymenoptera, Ichneumonidae). - Beiträge zur Entomologie 8: 438-477.

BAUER, R. 2002: Bemerkungen über die Ichneumoniden der Alpen. Teil IV (Hymenoptera, Ichneumonidae). - Entomofauna 23: 93-107.

Bengtsson, S. 1902: Ueber Pimpla capulifera Kriechb. (Hym.). - Zeitschrift für Systematische Hymenopterologie und Dipterologie 2: 369-372.

Betz, E. \& SChwerdTfeger, F. 1970: Lepidopteren-Parasiten aus zwei nordwestdeutschen Eichenwäldern. - Entomophaga 15: 347-351.

BouchÉ, P. F. 1834: Naturgeschichte der Insekten, besonders in Hinsicht ihrer ersten Zustände als Larven und Puppen. - Berlin: 1-216.

Brauns, S. 1898: Neue Schlupfwespen aus Mecklenburg. - Archiv des Vereins der Freunde der Naturgeschichte in Mecklenburg 51: 58-72.

Cole, L. R. 1959: On the defences of Lepidopterous pupae in relation to the oviposition behaviour of certain Ichneumonidae. - Journal of the Lepidopterists' Society 13: 1-10.

Dalla Torre, C. G. De 1902: Catalogus Hymenopterorum. Volume III. Trigonalidae, Megalyridae, Stephanidae, Ichneumonidae, Agriotypidae, Evaniidae, Pelecinidae. - Guilelmi Engelmann Lipsiae 1902: 545-1141.

Dathe, H. H.; Taeger, A. \& Blank, S. M. (Hrsg.) 2001: Verzeichnis der Hautflügler Deutschlands (Entomofauna Germanica 4). - Entomologische Nachrichten und Berichte, Beiheft 7: 1-178.

Drewsen, C. \& Boie, F. 1836: Beiträge zur Geschichte der Hymenopteren. - Archiv für Naturgeschichte 2: 35-44.

Ensuin, E. 1912-1918: Die Tenthredinoidea Mitteleuropas. - Deutsche Entomologische Zeitschrift, Berlin Beihefte 1-7 [1912-1917]: 1-790.

Fitton, M. G.; Shaw, M. R. \& Gauld, I. D. 1988: Pimpline Ichneumon-Flies. Hymenoptera, Ichneumonidae (Pimplinae). - Handbooks for the Identification of British Insects vol. 7, part 1: 1110; London.

GYÖrfI, J. 1963: Beiträge zur Biologie und Ökologie der Schlupfwespen (Ichneumonidae). - Zeitschrift für Angewandte Entomologie 51: 142-147. 
Habermenl, H. 1904: Beiträge zur Kenntnis der Ichneumoniden I. - Wissenschaftliche Beilage zum Jahresberichte des Grossh. Gymnasiums und der Oberrealschule zu Worms 1903/4: 37 S.

HabermeHL, H. 1917: Beiträge zur Kenntnis der palaearktischen Ichneumonidenfauna. - Zeitschrift für Wissenschaftliche Insektenbiologie 13: 110-117.

Haeselbarth, E. 1985: Determination list of entomophagous insects. 10. - International Union of Biological Sciences. International Organization for Biological Control of noxious animals and plants. WPRS Bulletin: $61 \mathrm{~S}$.

Haupt, H. 1913: Beiträge zur Hymenopteren-Fauna von Halle a. S. und Umgegend. - Mitteilungen der Entomologischen Gesellschaft Halle 5/7: 47-62.

Haupt, H. 1954: Fensterfänge bemerkenswerter Ichneumonen (Hym.), darunter 10 neuer Arten. - Deutsche Entomologische Zeitschrift 1: 99-116.

Hedwig, K. 1950: Ichneumoniden der Umgebung von Würzburg (einzelne von der Rhön u. Lohr). Nachrichten des Naturwissenschaftlichen Museums der Stadt Aschaffenburg 29: 43-56.

Hedwig, K. 1958: (Hym.) Schlupfwespen (Ichneumoniden) aus der Eysselheide von Gifhorn/Niedersachsen II. - Bombus 2: 31-34.

Hedwig, K. 1959: Entomologische Beobachtungen in der Braunschweiger Börde. Ein Beitrag zur Fauna der parasitisch lebenden Hymenopteren und ihrer Wirte. - Verhandlungen des Vereins für naturwissenschaftliche Heimatforschung 34: 32-47.

Heinrich, G. H. 1949: Ichneumoniden des Berchtesgadener Gebietes. (Hym.). - Mitteilungen der Münchener Entomologischen Gesellschaft 35-39: 1-101.

Hilpert, H. 1987a: Erster Beitrag zur Kenntnis der südbadischen Schlupfwespenfauna. Ichneumoniden des Feldberggebietes. I. Faunistik (Hymenoptera, Ichneumonidae). - Mitteilungen des Badischen Landesvereins für Naturkunde und Naturschutz 14: 343-360.

Hilpert, H. 1987b: Schlupfwespen des Feldberggebietes (Hymenoptera, Ichneumonidae). - Carolinea 45: 147-158.

Hilpert, H. 1989: Zur Hautflüglerfauna eines südbadischen Eichen-Hainbuchenmischwaldes. - Spixiana 12: $57-90$.

Horstmann, K. 1970: Die Ichneumoniden (Hymenoptera) von der Nordseeküste Schleswig-Holsteins. - Faunistisch-Ökologische Mitteilungen 3: 299-307.

Horstmann, K. 1971: Untersuchungen über die Parasiten der an Eichen lebenden Tortriciden während einer Massenvermehrung des Grünen Eichenwicklers (Tortrix viridana L.). - Waldhygiene 8: 226-242.

Horstmann, K. 1984: Untersuchungen zum Massenwechsel des Eichenwicklers, Tortrix viridana L. (Lepidoptera, Tortricidae), in Unterfranken. - Zeitschrift für Angewandte Entomologie 98: 73-95.

Horstmann, K. 1988: Die Schlupfwespenfauna der Nordsee-Inseln Mellum und Memmert (Hymenoptera, Ichneumonidae). - Drosera 88: 183-206.

Horstmann, K. 2001: Ichneumonidae. - In: Dathe, H. H.; Taeger, A. \& Blank, S. M. (Hrsg.): Verzeichnis der Hautflügler Deutschlands (Entomofauna Germanica 4). - Entomologische Nachrichten und Berichte, Beiheft 7: 69-103.

Horstmann, K. 2002: Bemerkungen zu einer Liste der aus Deutschland nachgewiesenen Ichneumonidae (Hymenoptera). - Nachrichtenblatt der Bayerischen Entomologen 51: 75-80.

Horstmann, K. \& Floren, A. 2001: Ichneumonidae aus den Baumkronen eines nordbayerischen Eichenhochwaldes (Insecta: Hymenoptera). - Beiträge zur Bayerischen Entomofaunistik 4: 209-214.

JäNneR, G. 1919a: Beitrag zur Verbreitung der Thüringer Pimpla. - Internationale Entomologische Zeitschrift 12: 182-184.

Jänner, G. 1919b: Beitrag zur Verbreitung der Thüringer Pimpla. - Internationale Entomologische Zeitschrift 12: 193-195. 
Kasparyan, D. R. 1973: A review of the Palearctic Ichneumonids of the tribe Pimplini (Hymenoptera, Ichneumonidae). The genera Itoplectis Foerst. and Apechthis Foerst. - Entomologicheskoye Obozreniye 52: 665-681.

Kasparyan, D. R. 1981: Sem. Ichneumonidae, Vvedenie, Podsemejstvo Pimplinae - In: G. S. Medvedev (ed.). Opredelitel nasekomykh evropeyskoy chasti SSSR, Tom III. Pereponchatokrylyye, tretya chast, p. 7-97; Nauka, Leningrad.

Kettner, F. W. 1968: Die Schlupfwespen (Ichneumonidae) Nordwestdeutschlands (II. Teil). - Verhandlungen des Vereins für naturwissenschaftliche Heimatforschung zu Hamburg 37: 51-90.

KLausnitzer, B. 2003: Gesamtübersicht zur Insektenfauna Deutschlands. - Entomologische Nachrichten und Berichte 47: 57-66.

Kolarov, J. A. 1997: Hymenoptera, Ichneumonidae. Part I. Pimplinae, Xoridinae, Acaenitinae, Collyriinae. - Fauna Bulgarica 25: 1-322.

Kolarov, J. 2004: New data on the structure of the flagellum in males of the genus Apechthis Foerster (Hymenoptera, Ichneumonidae, Pimplinae). - Linzer Biologische Beiträge 36: 265-271.

Kriechbaumer, J. 1887: Pimpliden-Studien. 6-10. - Entomologische Nachrichten 13: 113-121.

LANGE, C. 1898: Ergebnisse entomologischer Beobachtungen aus der Umgebung Annabergs. X - Berichte des Annaberg-Buchholzer Vereins für Naturkunde 1898: 53-104.

OentKe, J. 1964: Übersicht und Bestimmungstabelle der palaearktischen Gattungen der ehemaligen Unterfamilie Pimplinae auct. (Hymenoptera, Ichneumonidae). - Entomologische Abhandlungen 29: 533-590; Dresden.

Oенцке, J. 1967: Westpaläarktische Ichneumonidae I. Ephialtinae. - Hymenopterorum Catalogus (nova editio) 2: 1-49; 's-Gravenhage.

OenLKe, J. 1969: Beiträge zur Insektenfauna der DDR: Hymenoptera - Bestimmungstabellen bis zu den Unterfamilien. - Beiträge zur Entomologie 19: 753-801.

Pfankuch, K. 1925: Die von Norwich gesammelten paläarktischen Ichneumoniden des Bremer Museums. - Abhandlungen des Naturwissenschaftlichen Vereins zu Bremen 26: 37-58.

Pfankuch, K. 1935: Verzeichnis der Ichneumoniden von Bremen und Umgegend. - Mitteilungen aus dem Entomologischen Verein in Bremen 22 (1934): 6-30.

Pfeffer, W. 1913: Die Ichneumoniden Württembergs mit besonderer Berücksichtigung ihrer Lebensweise. I. Teil. - Jahresheft des Vereins für Vaterländische Naturkunde 69: 303-353.

Rudow, F. 1917: Ichneumoniden und ihre Wirte. - Entomologische Zeitschrift 31: 61-62.

SAWONIEWICZ, J. 1979: The effect of shrub layer on the occurence of the Ichneumonidae (Hymenoptera) in pine stands on different sites. - Memorabilia Zoologica 30: 89-130.

Schmiedeknecht, O. 1907: Opuscula Ichneumonologica. III. Band. (Fasc. XV-XVII.) Pimplinae. S. 1121-1360. Blankenburg in Thüringen.

Schmiedeknecht, O. 1914: Die Ichneumonidengattung Pimpla F. - Zeitschrift für Angewandte Entomologie 1: 369-478.

Schütze, K. T. \& Roman, A. 1931: Schlupfwespen. - Isis Budissina 12 (1928-1930): 3-12.

ŠEDIVÝ, J. 1963: Faunistische und taxonomische Bemerkungen zu den Ichneumoniden der Tschechoslowakei, Pimplinae, II. - Acta Faunistica Entomologica Musei Nationalis Pragae 9: 155-177.

Smits van Burgst, C. A. L. 1918: Naamlijst der in de Ichneumonen-Collectie van het rijk aanwezige genera en species der familie Ichneumonidae. - Luctor et Emergo 's Hage: $48 \mathrm{~S}$.

Starke, H. 1940: Erster Nachtrag zu den Schlupfwespen von K. T. Schütze, Rachlau und Dr. Roman, Stockholm. - Isis Budissina 14 (1936/1940): 63-95.

Starke, H. 1956: Ichneumonidenfauna der sächsischen Oberlausitz. - Natura Lusatica (Bautzen) 3: $17-92$.

Strand, E. 1913: Neue Beiträge zur Arthropodenfauna Norwegens. XX. Hymenoptera Parasitica. - Nytt Magasin for Naturvidenskapene 51: 337-361. 
Strand, E. 1918: Notizen zur Hymenopterenfauna Deutschlands. - Entomologische Zeitschrift 32: 7.

Uhthoff-Kaufmann, R. R. 1991: The distribution and occurence of the genus Saperda F. (Col.: Lamiidae) in Great Britain. - Entomologist's Record 103: 129-134.

Ulbricht, A. 1909: Beiträge zur Insekten-Fauna des Niederrheins. Ichneumoniden der Umgegend. Mitteilungen des Vereins für Naturkunde in Verbindung mit dem Städtischen Naturkundlichen Museum zu Krefeld 1909: 1-40.

Ulbricht, A. 1912: VIII. Pimplinae [aus Norwegen und Deutschland]. - Nytt Magasin for Naturvidenskapene 50: 23-28.

Ulbricht, A. 1926: Niederrheinische Ichneumoniden. 4. Nachtrag. - Mitteilungen des Vereins für Naturkunde in Verbindung mit dem Städtischen Naturkundlichen Museum zu Krefeld 1926: 1-30.

WALTER, S. 1991: Ichneumonidae (Hymenoptera) in immissionsgeschädigten Fichtenforsten des Erzgebirges. - Zoologische Jahrbücher Abteilung für Systematik, Ökologie und Geographie der Tiere 118: 449-464.

Yu, D. S. 1999: Interactive catalogue of World Ichneumonidae 1998. - Taxapad, Vancouver: 57 S. + CDROM.

Yu, D. S. \& Horstmann, K. 1997: A catalogue of world Ichneumonidae (Hymenoptera). - Memoirs of the American Entomological Institute 58: 1-1558.

Anschrift des Verfassers:

Dipl.-Biol. Hans-Joachim Jacobs

Dorfstraße 41

17495 Ranzin

E-Mail: hjjacobs@t-online.de
Subject editor:

Dr. A. TAEgER

\section{Besprechungen}

Amiet, F.; Herrmann, M.; Müller, A. \& Neumeyer, R.: Apidae 5. (Ammobates, Ammobatoides, Anthophora, Biastes, Ceratina, Dasypoda, Epeoloides, Epeolus, Eucera, Macropis, Melecta, Melitta, Nomada, Pasites, Tetralonia, Thyreus, Xylocopa). - Neuchatel: CSCF/Schweizerische Entomologische Gesellschaft, 2007. - (Fauna Helvetica 20). - 356 S., zahlr. Abb. u. Verbreitungskarten. - ISSN 1422-6367, ISBN 978 2-88414-032-4.

Die Bearbeitung der Schweizer Bienen-Fauna (1: Allgemeines, Apis, Bombus; Insecta Helvetica, Fauna 12, 1996; 2: Colletidae, Halictidae part.; Fauna Helvetica 4, 1999; Apidae 3: Halictus, Lasioglossum. Fauna Helvetica 6, 2001; 4: Megachilidae. Fauna Helvetica 9, 2004) wird in der 5. Folge mit den Melittidae (Dasypopda, Macropis, Melitta) und „übrigen“ Apidae fortgesetzt. Insgesamt werden 147 Arten über reich illustrierte Bestimmungsschlüssel erschlossen und in beiden Geschlechtern morphologisch charakterisiert. Die ganzseitige Vorstellung jeder Art schließt Verbreitungsangaben - für die Schweiz mit Karte und Flugzeiten - sowie ggf. bionomische Daten wie den Nestbau, den Blütenbesuch und die Wirtsbindung ein. Man erhält also alles das, was man benötigt, um die Art zu erkennen und einzuordnen. Wie zuvor, hat Felix Amiet wieder seine spezielle Sicht konsequent verfolgt und eigenständig verarbeitet. Lediglich bei der umfangreichen und schwierigen Gattung Nomada versicherte er sich der Mitwirkung des Spezialisten Maximilian Schwarz (Ansfelden). Geprüft und ergänzt haben die Koautoren. Neu ist die zeitliche Einordnung der Funddaten, als „aktuell“ gelten nur noch Belege nach 1990. Das Literaturverzeichnis ist leider mehr als sparsam gehalten, der Platz wird für die Dopplungen in Schrift und Bild infolge der Zweisprachigkeit französisch und deutsch benötigt. Die praktische Brauchbarkeit freilich ist davon unbeeinträchtigt. Mit diesem Buch rücken unausweichlich die Andrenidae ins Blickfeld. Man darf gespannt sein! 